\title{
Optimality Principles and Motion Planning of Human-Like Reaching Movements
}

\author{
Mikhail M. Svinin*, Igor A. Goncharenko**, \\ Shigeyuki Hosoe* and Yoshihito Osada* \\ ${ }^{*}$ The Institute of Physical and Chemical Research, RIKEN, Japan \\ **3D Incorporated, Yokohama, Japan
}

\begin{abstract}
The paper deals with modeling of human-like reaching movements. Several issues are under study. First, we consider a model of unconstrained reaching movements that corresponds to the minimization of control effort. It is shown that this model can be represented by the wellknown Beta function. The representation can be used for the construction of fractional order models and also for modeling of asymmetric velocity profiles. Next, we address the formation of boundary conditions in a natural way. From the mathematical point of view, the structure of the optimal solution is defined not only by the form of the optimality criterion but also by the boundary conditions of the optimization task. The natural boundary conditions, defined in this part of the paper, can also be used in modeling asymmetric velocity profiles. Finally, addressing the modeling of reaching movements with bounded control actions, we consider the minimum time formulation of the optimization problem and (for the $n$-th order integrator) find its analytical solution.
\end{abstract}

\section{Introduction}

Understanding the trajectory formation in reaching movements is a very important research problem in computational neuroscience and modern robotics. From the viewpoint of control theory the problem can be attacked from two directions, one deals with the open-loop control and employs optimization approaches (Flash et al., 2003; Kawato, 1999) while the other is based on the sensory-motor feedback control (Arimoto et al., 2005; Tsuji et al., 2002). These two research lines are quite different but both important because, in our opinion, it is the blending of the feedforward and feedback control that defines the appearance of reaching movements.

Indeed, experiments show that for relatively slow movements the velocity profiles are leftskewed (Milner and Ijaz, 1990; Nagasaki, 1989), a feature that is well-captured by the feedback control. On the other hand, for relatively fast movements the velocity profile of the hand trajectory tends to a symmetric ${ }^{1}$ bell-shaped form (Abend et al., 1982; Morasso, 1981), that can be captured by the feedforward control (Flash and Hogan, 1985). Thus, the resulting movement can be considered as the superposition of a major ballistic (feedforward) component and a

\footnotetext{
${ }^{1}$ Experiments also show that for very fast movements the velocity profiles can be even slightly skewed to the right (Nagasaki, 1989; Wiegner and Wierbicka, 1992).
} 
corrective (feedback) component (Berthier, 1996; Milner, 1992). How specifically the blending of these components works is one the greatest secrets of the CNS.

In this paper we will deal with the feedforward control of reaching movements. The reasoning behind this scheme is that in fast reaching movements the effective use of feedback is nearly impossible due to the delays in neural circuits and muscle activation. The feedforward control is often modeled via optimization theory. In the optimization approach, the trajectory of the human arm is predicted by minimizing, over the movement time $T$, an integral performance index $\mathcal{J}$ subject to boundary conditions imposed on start and end points. The performance index can be formulated in the joint space (Soechting and Lacquaniti, 1981; Uno et al., 1989) or in the task space normally associated with the human hand (Flash and Hogan, 1985; Ohta et al., 2004). Depending on whether the arm dynamics is taken into account or not, the performance index is defined in the motion (Flash and Hogan, 1985; Soechting and Lacquaniti, 1981) or force domain (Svinin et al., 2005; Uno et al., 1989).

Several modeling issues are under study in this paper. First, in Section 2 we consider a generalization of the classical minimum jerk model of the free-space reaching movements, relate it to the lowest polynomial approach, and show that the solution is given by the regularized incomplete Beta function. Next, in Section 3, we address the boundary conditions of the optimization task. Since the optimal solution is defined not only by the optimality criterion but also by the boundary conditions, the latter is not not less important than the former. However, the role of the boundary conditions is rarely discussed in the literature on reaching movements. Then, in Section 4, we address the formation of reaching movements with bounded control actions and consider the minimum time formulation of the optimization problem. Finally, conclusions are summarized in Section 5.

\section{Minimum effort model}

In our analysis, we will consider a simplified, one-dimensional model of human movements where the configuration dependence of the human arm is ignored and the motion considered at the hand level. For this model one can formulate a generalized criterion of optimality minimizing the squared $n$-th derivative of the hand position $x$ over the movement time:

$$
\mathcal{J}=\frac{1}{2} \int_{0}^{T}\left(\frac{d^{n} x}{d t^{n}}\right)^{2} d t
$$

From the control theoretical point of view this is the minimum effort criterion for the controlled system $x^{(n)}(t)=u$, describing the hand movement in the free space. Here, $u$ is the control input, and $n$ is the order of the motor dynamics associated with the hand. This order implies $2 n$ boundary conditions necessary for the correct formulation of the optimization problem. The state vector is composed of the hand position and its first $n-1$ derivatives. Assuming that the hand is at rest in the beginning and in the end of the reaching movement, one assigns

$$
\begin{aligned}
& x(0)=0, \quad \dot{x}(0)=0, \quad \ddot{x}(0)=0, \ldots, x^{(n-1)}(0)=0, \\
& x(T)=L, \quad \dot{x}(T)=0, \quad \ddot{x}(T)=0, \ldots, x^{(n-1)}(T)=0,
\end{aligned}
$$

where $L$ stands for the length of reaching movement.

The optimization problem-minimization of the criterion (1) under the boundary conditions $(2,3)$ - is often raised in the prediction of human reaching movements, normally for $n=3$ (the 
minimum hand jerk model (Flash and Hogan, 1985)) and occasionally for $n=4$ (the minimum snap model (Flash, 1983; Wiegner and Wierbicka, 1992)). Comparing to these models, the minimum hand acceleration model $(n=2)$ is often rejected on the ground that it produces non-zero initial and final accelerations, which is considered to be incompatible with experimental data (Stein et al., 1986).

A general formula for the optimal velocity profiles has been suggested in (Piazzi and Visioli, 2000; Richardson and Flash, 2002). Here, we present a formal derivation, establish the solution, and recognize it as a famous special mathematical function. To pose the optimization problem in non-dimensional settings, introduce the following change of variables: $x(t)=L y(\tau(t))$ where $\tau=t / T, \tau \in[0,1]$. In the new variables $y$ and $\tau$ we have the criterion

$$
\mathcal{J}=\frac{1}{2} \int_{0}^{1}\left(\frac{d^{n} y}{d \tau^{n}}\right)^{2} d \tau,
$$

and the boundary conditions

$$
\begin{aligned}
& y(0)=0, \quad y^{\prime}(0)=0, y^{\prime \prime}(0)=0, \ldots, y^{(n-1)}(0)=0, \\
& y(1)=1, \quad y^{\prime}(1)=0, y^{\prime \prime}(1)=0, \ldots, y^{(n-1)}(1)=0,
\end{aligned}
$$

where $y^{(k)}(\tau)=T^{k} x^{(k)}(t) / L, k=1, \ldots, n-1$. As can be easily shown, the Euler-Lagrange equation for the problem under consideration is $y^{(2 n)}(\tau)=0$. The general solution for this equation is given by the polynomial $y(\tau)=\sum_{i=0}^{2 n-1} c_{i} \tau^{i}$, where the coefficients $c_{i}$ are established from the boundary conditions $(5,6)$. Thus, the optimization problem under consideration is equivalent to the construction of the lowest order polynomial satisfying the boundary conditions $(5,6)$.

To clarify the analytical structure of the solution, we recast the optimization task as the optimal control problem. Let us introduce the state vector $\boldsymbol{y}=\left\{y, y^{\prime}, \ldots, y^{(n-1)}\right\}^{\mathrm{T}}$ and define the control input $u=y^{(n)}$. The state dynamics are the $n$-th order integrator $\dot{\boldsymbol{y}}=\boldsymbol{A} \boldsymbol{y}+\boldsymbol{b} u$, where $\boldsymbol{b}=\{0, \ldots, 0,1\}^{\mathrm{T}}$, and the elements of the matrix $\boldsymbol{A}$ are defined as

$$
[\boldsymbol{A}]_{i j}= \begin{cases}1 & \text { if } j=i+1 \\ 0 & \text { otherwise. }\end{cases}
$$

The analytical solution for the minimum effort control problem, seeking the control minimizing $\mathcal{J}=\frac{1}{2} \int_{0}^{1} u^{2} d \tau$ for the dynamic system $\dot{\boldsymbol{y}}=\boldsymbol{A} \boldsymbol{y}+\boldsymbol{b} u$ and the boundary conditions $\boldsymbol{y}(0)=\boldsymbol{y}_{0}, \boldsymbol{y}(1)=\boldsymbol{y}_{1}$, is well-established in the control literature (Luenberger, 1969). It can be represented as

$$
\boldsymbol{y}(\tau)=e^{\boldsymbol{A} \tau}\left(\left\{\boldsymbol{I}-\boldsymbol{W}(\tau) \boldsymbol{W}^{-1}(1)\right\} \boldsymbol{y}_{0}+\boldsymbol{W}(\tau) \boldsymbol{W}^{-1}(1) e^{-\boldsymbol{A}} \boldsymbol{y}_{1}\right)
$$

where

$$
\boldsymbol{W}(\tau)=\int_{0}^{\tau} e^{-\boldsymbol{A} s} \boldsymbol{b} \boldsymbol{b}^{\mathrm{T}} e^{-\boldsymbol{A}^{\mathrm{T}} s} d s
$$

For the $n$-th order integrator the matrix exponent is defined as

$$
\left[e^{\boldsymbol{A} \tau}\right]_{i j}=\left\{\begin{array}{cc}
\frac{\tau^{j-i}}{(j-i) !} & \text { if } j \geq i \\
0 & \text { if } j<i
\end{array}\right.
$$


and by direct calculations one finds

$$
[\boldsymbol{W}(\tau)]_{i j}=\frac{(-1)^{i+j} \tau^{2 n+1-i-j}}{(2 n+1-i-j)(n-i) !(n-j) !} .
$$

As shown in (Rose and Bronson, 1969), for the $n$-th order integrator the matrix $\boldsymbol{W}(\tau)$ can be decomposed as $\boldsymbol{W}(\tau)=\tau \boldsymbol{P}(-\tau) \boldsymbol{H P}(-\tau)$, where

$$
[\boldsymbol{P}(\tau)]_{i j}=\left\{\begin{array}{cl}
\frac{\tau^{n-j}}{(n-i) !} & \text { if } i=j \\
0 & \text { otherwise }
\end{array}\right.
$$

and $\boldsymbol{H}$ is the Hilbert matrix

$$
[\boldsymbol{H}]_{i j}=\frac{1}{(2 n+1-i-j)},
$$

the inverse of which is known to be

$$
\left[\boldsymbol{H}^{-1}\right]_{i j}=\frac{(-1)^{i+j}(2 n-i) !(2 n-j) !}{(2 n+1-i-j)\{(n-i) !(n-j) !\}^{2}(i-1) !(j-1) !} .
$$

The elements of the inverse matrix $\boldsymbol{W}^{-1}(\tau)=\tau^{-1} \boldsymbol{P}^{-1}(-\tau) \boldsymbol{H}^{-1} \boldsymbol{P}^{-1}(-\tau)$ can now be easily established:

$$
\left[\boldsymbol{W}^{-1}(\tau)\right]_{i j}=\frac{(2 n-i) !(2 n-j) !}{(2 n+1-i-j)(n-i) !(n-j) !(i-1) !(j-1) ! \tau^{2 n+1-i-j}} .
$$

For the rest-to-rest movements $\boldsymbol{y}_{0}=\mathbf{0}, \boldsymbol{y}_{1}=\{1,0 \ldots, 0\}^{\mathrm{T}}$, and $e^{-\boldsymbol{A}} \boldsymbol{y}_{1}=\boldsymbol{y}_{1}$. Therefore, $\boldsymbol{y}(\tau)=e^{\boldsymbol{A} \tau} \boldsymbol{W}(\tau) \boldsymbol{W}^{-1}(1) \boldsymbol{y}_{1}$. The components of the vector $\boldsymbol{y}(\tau)$, which are the derivatives of the corresponding order of $y(\tau)$, are found from $(10,11,15)$ by direct computation:

$$
y^{(i-1)}(\tau)=\sum_{j=i}^{n} \sum_{s=1}^{n} \frac{(-1)^{j+s}(2 n-1) !(2 n-s-1) ! \tau^{2 n+1-i-s}}{(j-i) !(2 n+1-s-j)\{(n-s) !\}^{2}(n-j) !(n-1) !(s-1) !} .
$$

To find the velocity $y^{\prime}(\tau)$, we set $i=2$ in (16) and take into account that

$$
\begin{aligned}
\sum_{j=2}^{n} \frac{(-1)^{j}}{(j-2) !(2 n+1-s-j)(n-j) !} & =-\frac{1}{(s-n-1)(s-n-2) \ldots(s-n-(n-1))} \\
& =\frac{(-1)^{n} \Gamma(1+n-s)}{\Gamma(2 n-s)}=\frac{(-1)^{n}(n-s) !}{(2 n-s-1) !}
\end{aligned}
$$

where $\Gamma$ is the usual Gamma function. Then, after some simple manipulations using the binomial expansion for $(1-\tau)^{n-1}$, one obtains

$$
y^{\prime}(\tau)=\frac{(2 n-1) ! \tau^{n-1}}{(n-1) !} \sum_{s=1}^{n} \frac{(-1)^{n+s} \tau^{n-s}}{(n-s) !(s-1) !}=\frac{\tau^{n-1}(1-\tau)^{n-1}}{B(n, n)}
$$

where

$$
B(n, n)=\frac{(n-1) !(n-1) !}{(2 n-1) !}
$$


is the symmetric Beta function. Therefore, the solution $y(\tau)$ can be formally represented as

$$
y(\tau)=\frac{\int_{0}^{\tau} p^{n-1}(1-p)^{n-1} d p}{B(n, n)} \triangleq \frac{B(\tau ; n, n)}{B(n, n)} \triangleq \bar{B}(\tau ; n, n) .
$$

This expression is known as the regularized incomplete Beta function (Abramowitz and Stegun, 1972). While it is widely used in many fields, it is best known for its applications in statistics (Dutka, 1981). The basic properties of the Beta distribution are well-known (Abramowitz and Stegun, 1972). In particular, the velocity $y^{\prime}(\tau)$ is symmetric with respect to the middle point $\tau=1 / 2$, the position $y(1 / 2)=1 / 2$, and all even derivatives $y^{(2 k)}(1 / 2)=0$ for $k=1,2,3 \ldots$.
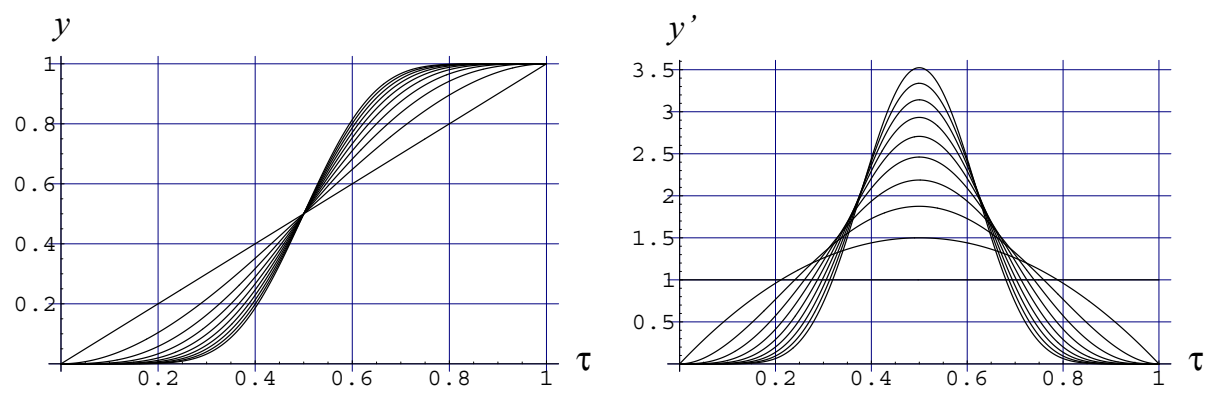

Fig. 1. Normalized position and velocity profiles in the minimum effort model for $n=$ $1,2, \ldots, 10$.

The first 10 solutions for $y(\tau)$ and $y^{\prime}(\tau)$ are plotted in Figure 1. While the solutions $y_{n}(\tau)$ are continuous functions of $\tau$ for any fixed $n$, in the limiting case of $n \rightarrow \infty$ the solution is discontinuous. In this case the velocity is the unit impulse function $y_{\infty}^{\prime}(\tau)=\delta(\tau-1 / 2)$, $\int_{0}^{1} y_{\infty}^{\prime}(\tau) d \tau=1$. The position $y_{\infty}(\tau)$ is the unit step function, which is unity for $\tau>1 / 2$ and is 0 for $\tau<1 / 2$.

Several comments are in order.

$1^{\mathrm{O}}$. It is noticed in (Flash and Hogan, 1985) that while, in general, the minimum jerk model $(n=3)$ is a good candidate for mimicking human-like movements, the minimum snap model $(n=4)$ sometimes also provide a reasonable fit to the experimental data of unconstrained reaching movements. In this connection, it should be noted that the Beta function is defined not only for integer but also for real $n$. This suggests that the criterion (1) can be generalized to non-integer orders using, for example, Riemann-Liouville fractional integrals and derivatives (Samko et al., 1993). It also suggests that in fitting experimental data of reaching movements we can use real numbers $n \in[3,4]$ (see Figure 2). The assumption of real $n$ sounds biologically plausible because the natural sensors and actuators in the human body are likely to deal with the fractional derivatives.

$2^{\mathrm{O}}$. The symmetry of the Beta function with respect to its last two arguments in $(18,20)$ is explained by the symmetric placement of the boundary conditions. It can be shown that for the asymmetric placement $\left(n_{a}\right.$ boundary conditions at the start point and $n_{b}$ at the end point, $\left.n_{a}+n_{b}=2 n\right)$ the solution is expressed through the asymmetric Beta function

$$
y(\tau)=\frac{\int_{0}^{\tau} p^{n_{a}-1}(1-p)^{n_{b}-1} d p}{B\left(n_{a}, n_{b}\right)} \triangleq \frac{B\left(\tau ; n_{a}, n_{b}\right)}{B\left(n_{a}, n_{b}\right)}
$$



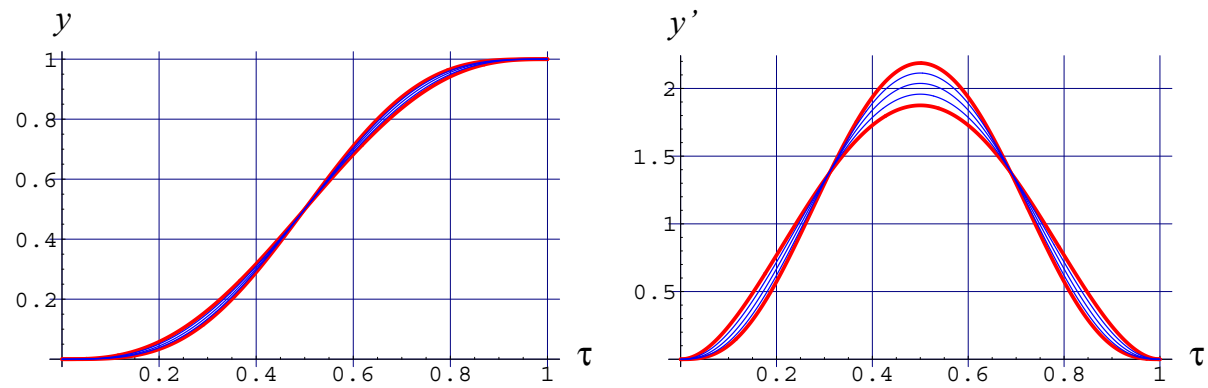

Fig. 2. Normalized position and velocity profiles for the integer (red lines: $n=3$ and $n=4$ ) and fractional (blue lines: $n=3.25, n=3.5$, and $n=3.75$ ) orders.

where

$$
B\left(n_{a}, n_{b}\right)=\frac{\left(n_{a}-1\right) !\left(n_{b}-1\right) !}{\left(n_{a}+n_{b}-1\right) !} .
$$

The asymmetric Beta functions are illustrated in Figure 3.
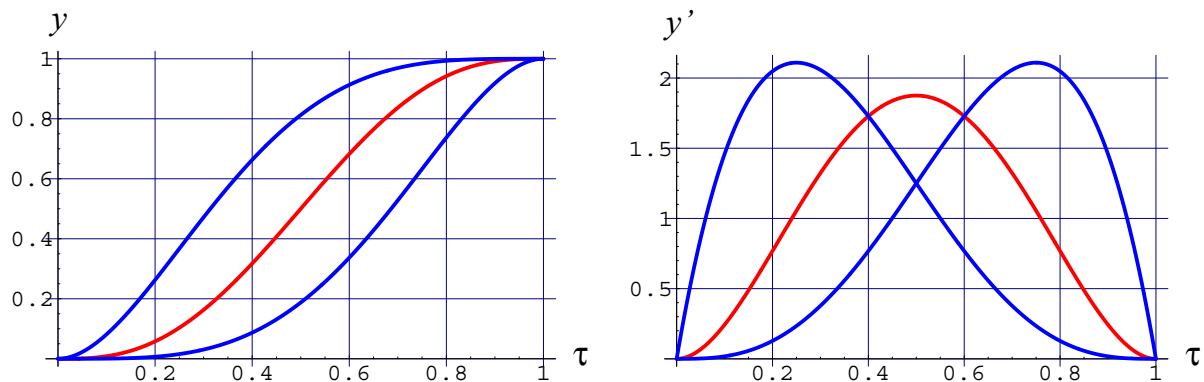

Fig. 3. Normalized position and velocity profiles given by the symmetric (red line: $B(3,3)$, center) and asymmetric (blue lines: $B(2,4)$ on the left, and $B(4,2)$ on the right) Beta functions.

Note that while the function (21) is the lowest order polynomial satisfying the asymmetric boundary conditions, it does not have a variational meaning. Strictly speaking, it does not minimize (4) because in the classical variational formulation the boundary conditions at the end-points (those that are imposed directly, as in $(5,6)$, and those obtained automatically from the variational formulation and sometimes called the natural boundary conditions (Gelfand and Fomin, 1963)) are always placed symmetrically.

\section{Natural boundary conditions}

This section addresses the formation of the boundary conditions. They are an important part of the optimization problem because the optimal solution is defined not only by the form of the optimality criterion but also by the boundary conditions, and they are not less important than the criterion itself.

In the optimization problem considered in Section 2 we fixed first $n-1$ derivatives of the hand position, which, as far as human movements are concerned, might not be always plausible 
from the physiological point of view ${ }^{2}$. Note that in experiments human subjects are normally not literally requested to produce zero end-point accelerations; they are only instructed to move comfortably from one point to another and stop there. Therefore, it does not seem reasonable to constrain the initial and final hand accelerations to zero in the optimization models. We can reliably pose the boundary conditions only for position and velocities. The remaining boundary conditions should be established differently.

Consider again the optimality criterion given by (1) but assume now that instead of $(2,3)$ we fix only the first $k \leq n-1$ derivatives of the hand position:

$$
\begin{array}{lll}
x(0)=0, & \dot{x}(0)=0, \ldots, & x^{(k)}(0)=0, \\
x(T)=L, & \dot{x}(T)=0, \ldots, & x^{(k)}(T)=0 .
\end{array}
$$

The variation of the functional (1) is defined as

$$
\delta \mathcal{J}=\int_{0}^{T} x^{(2 n)}(t) \delta x d t+\left[\sum_{i=0}^{n-1} x^{(2 n-1-i)}(t) \delta x^{(i)}(t)\right]_{0}^{T}
$$

Taking into account that $\delta x^{(i)}(0)=\delta x^{(i)}(T)=0$ for $i=0, \ldots, k$, from the necessary condition of optimality, $\delta \mathcal{J}=0$, one obtains the Euler-Lagrange equation $x^{(2 n)}(t)=0$, and establishes the following boundary conditions:

$$
\begin{aligned}
& x^{(n)}(0)=0, \quad x^{(n+1)}(0)=0, \ldots, \quad x^{(2 n-2-k)}(0)=0, \\
& x^{(n)}(T)=0, \quad x^{(n+1)}(T)=0, \ldots, \quad x^{(2 n-2-k)}(T)=0,
\end{aligned}
$$

Note that in the calculus of variations the boundary conditions $(26,27)$, obtained from the condition $\delta \mathcal{J}=0$, are called natural (Gelfand and Fomin, 1963). The solution of the optimization problem is the polynomial of degree $2 n-1$ whose coefficients are established from the directly imposed $(23,24)$ and the natural $(26,27)$ boundary conditions.

Consider, for the purpose of illustration, the case of $n=3$ and $k=1$. In this model we fix the boundary conditions for the position and velocities of the hand, while the hand accelerations in the beginning and the end of movement are defined Şautomatically $\breve{T}$ from the minimization of the squared hand jerk over the movement time. This differs from the conventional formulation where the boundary conditions for accelerations are set to zero. The minimum hand jerk model with the boundary conditions $(23,24)$ and $(26,27)$ yields

$$
x=L \tau^{2}\left(2.5-2.5 \tau^{2}+\tau^{3}\right),
$$

where $\tau=t / T$, while the conventional minimum hand jerk model gives

$$
x=L \tau^{3}\left(10-15 \tau+6 \tau^{2}\right) .
$$

Both these models produce single-phased velocity profiles. However, the maximal velocity in the former model is $1.5625 \mathrm{~L} / \mathrm{T}$, while in the latter one it is $1.875 \mathrm{~L} / \mathrm{T}$.

It should be noted that the solution (28) features non-zero initial and final acceleration jumps because in this model $\ddot{x}(0) \neq 0$ and $\ddot{x}(T) \neq 0$. To avoid possible confusion, it should be said that no coordinate jumps are physical, of course. Strictly speaking, the exact modeling of this

\footnotetext{
${ }^{2}$ For example, in the minimum snap model $(n=4)$ the end-point jerk are supposed to be zero. However, it is not evident that human can do the end-point control of the 3rd derivative of the hand position.
} 
phenomenon requires more sophisticated models considered at different time scales as the coordinate (acceleration, in our case) change is considerably high in a short instance of time. However, in simplified models, such as the one considered in our paper, this behavior can be modeled by the jumps in the boundary conditions.

The assumption of zero initial and final hand accelerations can be too restrictive when dealing with fast movements in highly dynamic reaching tasks Svinin et al. (2008). For the free-space movements the applicability of the models (28) or (29) is likely to depend on the movement duration $T$. For relatively slow movements the CNS has enough time to nullify the end-point accelerations and this defines the model (29). For relatively fast movements the end-point accelerations are placed at will and one gets the model (28).

To unify the minimum hand jerk model (28) with natural boundary conditions for accelerations with the conventional one (29), it is reasonable to assume that the CNS controls the boundary conditions. Intuitively, the way they are controlled is changed from task to task and, in general, the more dynamic the reaching task the looser the control of the boundary conditions.

To reflect the cost of control of the boundary conditions, one can replace the optimality criterion (1) by

$$
\mathcal{J}=\frac{1}{2} \int_{0}^{T}\left(\frac{d^{n} x}{d t^{n}}\right)^{2} d t+\frac{1}{2} \sum_{i=0}^{n-1} w_{i 0}\left(x^{(i)}(0)-\bar{x}_{0}^{i}\right)^{2}+w_{i T}\left(x^{(i)}(T)-\bar{x}_{T}^{i}\right)^{2} .
$$

Here, $\bar{x}_{0}^{i}$ and $\bar{x}_{T}^{i}$ are the "desired" steady state values for the derivatives of the hand trajectory at the boundary points, and $w_{i 0} \geq 0$ and $w_{i T} \geq 0$ are the corresponding weight coefficients assigned by the CNS. For simplicity, we assume that $\bar{x}_{0}^{i}=0$ for $i=0, \ldots, n, \bar{x}_{T}^{i}=0$ for $i=1, \ldots, n$, and $\bar{x}_{T}^{0}=L$.

Consider now the criterion of optimality given by (30). Note that now the separation of the boundary conditions into the directly imposed and the natural ones is no longer necessary, as we assume that all of them are controlled by the CNS and all of them are established from the condition $\delta \mathcal{J}=0$. In other words, all the boundary conditions are natural now. It is straightforward to show that the variation of the functional (30) is defined as

$$
\begin{aligned}
\delta \mathcal{J}=\int_{0}^{T} x^{(2 n)}(t) \delta x d t+ & {\left[\sum_{i=0}^{n-1} x^{(2 n-1-i)}(t) \delta x^{(i)}(t)\right]_{0}^{T} } \\
& +\sum_{i=0}^{n-1} w_{i 0}\left(x^{(i)}(0)-\bar{x}_{0}^{i}\right) \delta x^{i}(0)+w_{i T}\left(x^{(i)}(T)-\bar{x}_{0}^{i}\right) \delta x^{i}(T) .
\end{aligned}
$$

From $\delta \mathcal{J}=0$ one obtains the Euler-Lagrange equation, $x^{(2 n)}(t)=0$, and establishes the natural boundary conditions. Since the variations $\delta x^{i}(t)$ are not restricted at the boundary points, one obtains:

$$
\begin{aligned}
& -x^{(2 n-1-i)}(0)+w_{i 0}\left(x^{(i)}(0)-\bar{x}_{0}^{i}\right)=0, \\
& x^{(2 n-1-i)}(T)+w_{i T}\left(x^{(i)}(T)-\bar{x}_{T}^{i}\right)=0,
\end{aligned}
$$

where $i=0, \ldots, n-1$. It is worth pointing out two limiting cases. First, if $w_{i 0} \rightarrow \infty$ (the CNS places a very high gain) the initial state for the $i$-th derivative coincides with the desired one, 
as the condition (32) is reduced to $x^{(i)}(0)=\bar{x}_{0}^{i}$. If, on the other hand, the CNS cannot afford to control $x^{(i)}(0)$ then $w_{i 0} \rightarrow 0$ and we get $x^{(2 n-1-i)}(0)=0$.

Let us continue the illustrative example of $n=3$ and assign $\bar{x}_{0}^{i}=0, i=0,1,2, \bar{x}_{T}^{0}=L$, and $\bar{x}_{T}^{i}=0, i=1,2$. Assuming the tight control for the initial and final position and velocity, we set $w_{i 0}=w_{i T}=\infty, i=0,1$. By considering, for simplicity, the symmetric assignment of the accelerations weights, define $w \triangleq w_{20}=w_{2 T}$. It is not difficult to show that the optimal solution, parameterized by the acceleration weight $w$ is defined as

$$
x=\frac{L \tau^{2}\left(30+10 \bar{w} \tau-15(2+\bar{w}) \tau^{2}+6(2+\bar{w}) \tau^{3}\right)}{(12+\bar{w})},
$$

where $\tau=t / T$, and $\bar{w}=w T$. For $w=0$ we get the model (28) with natural boundary conditions for accelerations, and for $w \rightarrow \infty$ we get the conventional minimum hand jerk model (29). Changing the acceleration weight from zero to infinity defines a family of curves (all in the class of the minimum hand jerk models). The velocity and acceleration profiles of this family for $L=1, T=1$ are shown in Fig. 4.
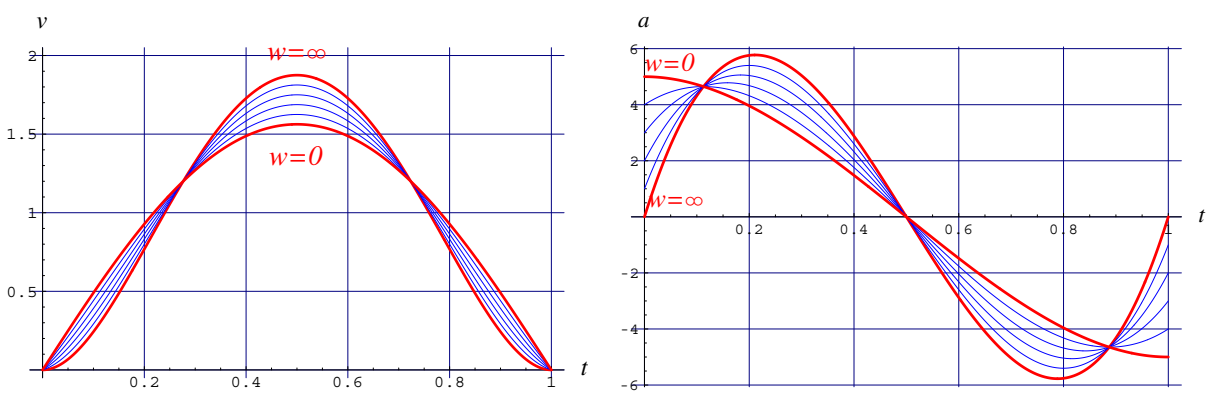

Fig. 4. Velocity and acceleration profiles for the family of curves (34).

Several comments are in order.

$1^{\mathrm{O}}$. Asymmetric velocity profiles can be obtained by asymmetric placement of the weight coefficients $\left(w_{i 0} \neq w_{i T}\right)$. Note also that the optimality criterion (30) can be generalized by the introduction of matrix weight coefficients.

$2^{\mathrm{O}}$. It is not clear how specifically the CNS may assign the weight coefficients. Intuitively, the human perception and control of position is better than that of velocity, that of velocity is better than that of accelerations, and so on, and it is plausible to assume that $w_{i, 0}>w_{i+1,0}$ and $w_{i, T}>w_{i+1, T}$. The specific assignment of the weight coefficients must depend on how fast the reaching movement under inspection is. In this connection, it should be noted that the analysis of reaching movements is often conducted under the subtle assumption that the CNS operates without undue stress, away from the limits of neuromuscular performance (Flash and Hogan, 1985). But this assumption is not specific.

It is reported that for comfortable reaching movements in free space the movement time is in the range of $[0.25,0.8] \mathrm{s}$ for the traveling distance varying from $0.2 \mathrm{~m}$ to $0.5 \mathrm{~m}$ (Kawato, 1995). Since the conventional minimum hand jerk model and the minimum torque change model are well established and experimentally verified for these ranges, one can presume that in such movements the boundary conditions for the hand accelerations are zero. However, if the movement time is decreased the CNS obviously cannot afford to keep the initial hand 
accelerations zero. Therefore, for curtain ranges of time the reaching movements will feature the acceleration jumps (soft impacts). And if the movement duration is decreased further, the movements will feature even the velocity jumps (hard impact). One can see such impulsive movements in the exercises of karate fighters.

Obviously, the movement time $T$ cannot be reduced arbitrarily without violating the constraints imposed by the neural and musculoskeletal hardware on the traveling distance, maximal force, and the accuracy of reaching. These constraints are ignored in the optimization problems considered in this section. Taking into account additional considerations would lead to different models with possibly different criteria of optimality (Nelson, 1983). One of these models is the minimum time model.

\section{Minimum time model}

In this section, the hand trajectory $x(t)$ is determined by minimizing the performance index

$$
\mathcal{J}=\int_{0}^{T} d t=T
$$

where $T$ is the movement time to be obtained. For the simplicity of formulation, we will consider the rest-to-rest time-optimal control where the boundary conditions are defined by $(2,3)$ and the scalar control input is bounded as

$$
|u(t)| \leq U
$$

For a linear controllable system, existence and uniqueness of the time-optimal solution are guaranteed, and the solution is "bang-bang" control with a finite number of switches (Hermes and LaSalle, 1969; Lee and Markus, 1967). It is easy to show that for the system under consideration ( $n$-th order integrator) the optimal solution is symmetric about $T / 2$, and the number of switches is $n+1\left(t_{0}=0, t_{1}, t_{2}, \ldots, t_{n}=T\right)$.

Assuming $L>0$, we set $u(t)=(-1)^{s} U$ for the time interval $t \in\left[t_{s}, t_{s+1}\right], s=0,1, \ldots, n-1$, and, by integrating $x^{(n)}=u(t)$, define the $(n-k)$-th derivative $(k=1, \ldots, n)$ of the hand position:

$$
x^{(n-k)}(t)=\frac{\left(t-t_{s}\right)^{k}}{k !}(-1)^{s} U+\sum_{p=0}^{k-1} x^{(n-k+p)}\left(t_{s}\right) \frac{\left(t-t_{s}\right)^{p}}{p !} .
$$

For $t=t_{s+1}$ one therefore has

$$
x^{(n-k)}\left(t_{s+1}\right)=\frac{\left(t_{s+1}-t_{s}\right)^{k}}{k !}(-1)^{s} U+\sum_{p=0}^{k-1} x^{(n-k+p)}\left(t_{s}\right) \frac{\left(t_{s+1}-t_{s}\right)^{p}}{p !} .
$$

By considering this recurrent relationship with the zero initial condition (2), one can represent $x^{(n-k)}\left(t_{s}\right)$ in the following non-recurrent form

$$
x^{(n-k)}\left(t_{s}\right)=\frac{U}{k !} \sum_{i_{1}=1}^{s} \sum_{i_{2}=1}^{s} \ldots \sum_{i_{k}=1}^{s}(-1)^{\min \left\{i_{1}, i_{2}, \ldots, i_{k}\right\}-1} \Delta t_{i_{1}} \Delta t_{i_{2}} \ldots \Delta t_{i_{k}}
$$


where $\Delta t_{i}=t_{i}-t_{i-1}$, and $s=1,2, \ldots, n$. Now, substitute $x^{(n-k)}\left(t_{n}\right), k=1,2, \ldots, n$, into the boundary condition (3). After some algebra, one obtains the following system of $n$ equations

$$
\begin{aligned}
& 2\left\{\sum_{i=1}^{n-1}(-1)^{i} t_{i}^{k}\right\}+(-1)^{n} t_{n}^{k}=0, k=1, \ldots, n-1, \\
& 2\left\{\sum_{i=1}^{n-1}(-1)^{i} t_{i}^{n}\right\}+(-1)^{n} t_{n}^{n}=(-1)^{n} n ! L / U,
\end{aligned}
$$

with respect to the unknown switching times $t_{1}, t_{2}, \ldots, t_{n}$. The system (40) is homogenous, and with the introduction of the non-dimensional times $\tau_{i}=t_{i} / t_{n}$ it is converted to the following form

$$
\sum_{i=1}^{n-1}(-1)^{i} \tau_{i}^{k}=(-1)^{n+1} / 2, \quad k=1, \ldots, n-1 .
$$

Let us show that the solution for this system is given by

$$
\tau_{i}=\sin ^{2}\left(\frac{\pi i}{2 n}\right), \quad i=1, \ldots, n-1 .
$$

First, by converting (43) to the double angle representation and substituting it into (42), one obtains

$$
\begin{aligned}
\sum_{i=1}^{n-1}(-1)^{i} \tau_{i}^{k}=\sum_{i=1}^{n-1}(-1)^{i}\left\{\frac{1}{2}\left(1-\cos \frac{\pi i}{n}\right)\right\}^{k}= & \\
= & \sum_{i=1}^{n-1}(-1)^{i} \frac{1}{2^{k}}\left(1-\cos \frac{\pi i}{n}\right)^{k}=\frac{1}{2^{k}} \sum_{j=0}^{k}(-1)^{j} C_{k}^{j} \sum_{i=1}^{n-1}(-1)^{i} \cos ^{j} \frac{\pi i}{n},
\end{aligned}
$$

where $C_{p}^{q}=p ! /(q !(p-q) !)$ denotes the binomial coefficient. Next, it is known (see section 4.4.2 of (Prudnikov et al., 1986)) that

$$
\sum_{k=0}^{n-1}(-1)^{k} \cos ^{m} \frac{\pi k}{n}=\left\{\begin{array}{cl}
\frac{1}{2}\left\{1-(-1)^{m+n}\right\} & \text { for } m=0,1,2, \ldots, n-1 \\
\frac{n}{2^{n-1}} & \text { for } m=n
\end{array} .\right.
$$

Therefore, for $k<n$ we have

$$
\begin{gathered}
\sum_{i=1}^{n-1}(-1)^{i} \tau_{i}^{k}=\frac{1}{2^{k}} \sum_{j=0}^{k}(-1)^{j} C_{k}^{j}\left\{\frac{1}{2}\left[-1-(-1)^{n+j}\right]\right\}= \\
=\frac{1}{2^{k+1}} \underbrace{\sum_{j=0}^{k} C_{k}^{j}(-1)^{j+1}}_{0}-\frac{(-1)^{n}}{2^{k+1}} \underbrace{\sum_{j=0}^{k} C_{k}^{j}}_{2^{k}}=-\frac{(-1)^{n}}{2} .
\end{gathered}
$$

Thus, the non-dimensional switching times $\tau_{i}$ are defined by (43). It remains now to find the duration of movement $T \triangleq t_{n}$. For this purpose, rewrite (41) as

$$
2\left\{\sum_{i=1}^{n-1}(-1)^{i} \tau_{i}^{n}\right\}+(-1)^{n}=(-1)^{n} n ! \frac{L}{U T^{n}} .
$$


By considering (44) for $k=n$ and taking into account (45) and (46), one obtains

$$
\begin{aligned}
& \sum_{i=1}^{n-1}(-1)^{i} \tau_{i}^{n}=\frac{1}{2^{n}} \sum_{j=0}^{n}(-1)^{j} C_{n}^{j} \sum_{i=1}^{n-1}(-1)^{i} \cos ^{j} \frac{\pi i}{n}= \\
& \underbrace{\frac{1}{2^{n}} \sum_{j=0}^{n-1}(-1)^{j} C_{n}^{j} \sum_{i=1}^{n-1}(-1)^{i} \cos ^{j} \frac{\pi i}{n}}_{-\frac{(-1)^{n}}{2}}+\frac{(-1)^{n}}{2^{n}} \underbrace{\sum_{i=1}^{n-1}(-1)^{i} \cos ^{n} \frac{\pi i}{n}}_{\frac{n}{2^{n-1}}}= \\
&-\frac{(-1)^{n}}{2}+\frac{(-1)^{n} n}{2^{2 n-1}} .
\end{aligned}
$$

Therefore, the minimal movement time is defined as

$$
T=\sqrt[n]{2^{2(n-1)}(n-1) ! L / U}
$$

and the control switching times $t_{0}, t_{1}, t_{2}, \ldots, t_{n}$ are computed as

$$
t_{i}=\sqrt[n]{\frac{2^{2(n-1)}(n-1) ! L}{U}} \sin ^{2}\left(\frac{\pi i}{2 n}\right), \quad i=0, \ldots, n .
$$
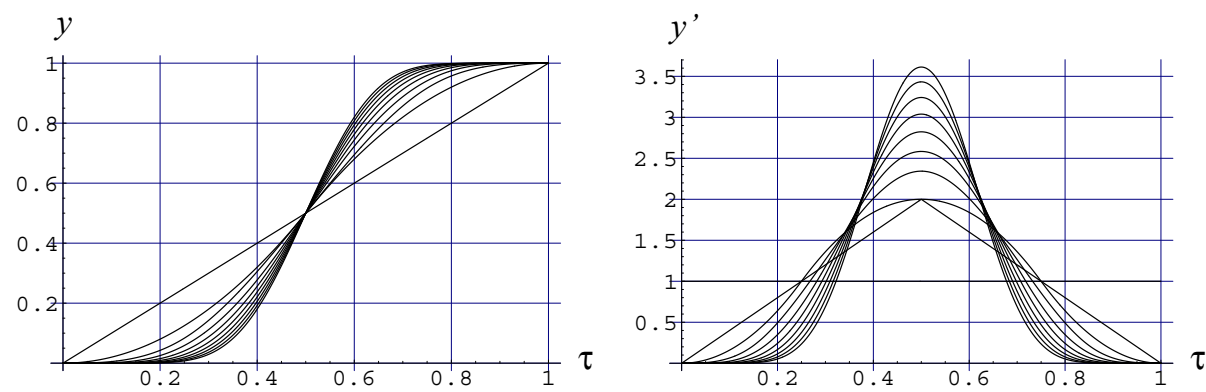

Fig. 5. Normalized position and velocity profiles in the minimum time model for $n=$ $1,2, \ldots, 10$.

The first 10 solutions for the normalized position and velocity profiles in the minimum time model are plotted in Figure 5. Comparing these plots with those shown in Figure 1, one can say that qualitatively the minimum time model is similar to the minimum effort model.

Several comments are in order.

$1^{\mathrm{O}}$. It should be noted that in modeling of rapid reaching movements the minimum time model is often rejected (in favor of, say, the minimum hand jerk model) on the ground that it features acceleration jumps, which is not compatible with experimental data (Stein et al., 1986; Wiegner and Wierbicka, 1992). The rejection of the minimum time model only for this reason is premature because it is based on the fallacious comparison of the minimum time model for $n=2$ with the minimum effort model for $n=3$.

$2^{\mathrm{O}}$. The minimum time model for $n=3$ does not feature acceleration jumps (the discontinuities here are at the jerk level). The velocity profile in this model looks like the typical 
bell-shaped pattern observed in experiments, with the normalized maximal velocity being between those in the minimum hand jerk $(n=3)$ and snap $(n=4)$ models (see Table 1$)$. Thus, the minimum time model for $n=3$ can be a legitimate candidate for mimicking human-like reaching movements.

\begin{tabular}{|c||c|c|c|c|c|c|c|c|}
\hline model & $n=1$ & $n=2$ & $n=3$ & $n=4$ & $n=5$ & $n=6$ & $n=7$ & $n=8$ \\
\hline \hline minimum effort & 1.0 & 1.5 & 1.875 & 2.188 & 2.461 & 2.707 & 2.933 & 3.142 \\
\hline minimum time & 1.0 & 2.0 & 2.0 & 2.343 & 2.584 & 2.823 & 3.039 & 3.242 \\
\hline
\end{tabular}

Table 1. Normalized velocity at the middle point: $\frac{L}{T} v\left(\frac{T}{2}\right)$.

$3^{\circ}$. An interesting approach to modeling of human-like reaching movements is proposed in (Ben-Itzhak and Karniel, 2008). The approach is based on the minimum acceleration model and can be linked to the minimization of kinetic energy. In the generalized form, for the controlled system $d^{n} x / d t^{n}=u(t)$ the trajectory is found by minimizing the criterion $\mathcal{J}=$ $\frac{1}{2} \int_{0}^{T}\left(d^{n-1} x / d t^{n-1}\right)^{2} d t$ under the constraints $|u(t)| \leq U$. This is a singular optimal control problem. We conjecture that this approach is equivalent to the minimum time model when the movement duration is specified by (49). This fact can be easily verified for $n=2$ and $n=3$ but remains to be proven for the general case.

\section{Conclusions}

Several issues related to modeling human-like reaching movements were under study in this paper. First, a mathematical model, based on the minimization of square derivatives of the hand position over the movement duration, has been established in the analytical form. This is a generalized model, described by the well-known Beta function. The representation can be used for the construction of fractional order models and also for modeling of asymmetric velocity profiles. Next, we addressed a natural (from the viewpoint of the calculus of variations) formation of the boundary conditions. From the mathematical point of view, the structure of the optimal solution is defined not only by the form of the optimality criterion but also by the boundary conditions of the optimization task. The natural boundary conditions, defined in our paper, can also be used in modeling asymmetric velocity profiles. Finally, addressing modeling reaching movements with bounded control actions, we have considered the minimum time formulation of the optimization problem and (for the $n$-th order integrator) established its analytical solution.

\section{References}

Abend, W., Bizzi, E., and Morasso, P. (1982). Human arm trajectory formation. Brain, 105:331348.

Abramowitz, M. and Stegun, I. (1972). Handbook of Mathematical Functions With Formulas, Graphs and Mathematical Tables. U.S. Government Printing Office, Washington D.C., 10th edition.

Arimoto, S., Sekimoto, M., Hashiguchi, H., and Ozawa, R. (2005). Natural resolution of illposedness of inverse kinematics for redundant robots: A challenge to bernstein's degrees-of-freedom problem. Advanced Robotics, 19(4):401-434. 
Ben-Itzhak, S. and Karniel, A. (2008). Minimum acceleration criterion with constraints implies bang-bang control as an underlying principle for optimal trajectories of arm reaching movements. Neural Computation, 20(3):779-812.

Berthier, N. (1996). Learning to reach: A mathematical model. Developmental Psychology, 32(5):811-823.

Dutka, J. (1981). The incomplete Beta function-a historical profile. Archive for History of Exact Sciences, 24:11-29.

Flash, T. (1983). Organizing Principles Underlying The Formation of Hand Trajectories. PhD thesis, Massachusetts Institute of Technology, Cambridge, USA.

Flash, T. and Hogan, N. (1985). The coordination of arm movements: An experimentally confirmed mathematical model. The Journal of Neuroscience, 5(7):1688-1703.

Flash, T., Hogan, N., and Richardson, M. (2003). Optimization principles in motor control. In Arbib, M., editor, The Handbook of Brain Theory and Neural Networks, pages 827-831. MIT Press, Cambridge, Massachusetts, 2nd edition.

Gelfand, I. and Fomin, S. (1963). Calculus of Variations. Prentice-Hall, Englewood Cliffs, New Jersey.

Hermes, H. and LaSalle, J. (1969). Functional Analysis and Time-Optimal Control. Academic, New York.

Kawato, M. (1995). Motor learning in the brain. Journal of the Robotics Society of Japan, 13(1):1119.

Kawato, M. (1999). Internal models for motor control and trajectory planning. Current Opinion in Neurobiology, 9:718-727.

Lee, E. and Markus, L. (1967). Foundations of Optimal Control Theory. Wiley, New York.

Luenberger, D. (1969). Optimization by Vector Space Methods. John Wiley \& Sons, New York.

Milner, T. (1992). A model for the generation of movements requiring endpoint precision. Neuroscience, 49(2):487-496.

Milner, T. and Ijaz, M. (1990). The effect of accuracy constraints on three-dimensional movement kinematics. Neuroscience, 35(2):365-374.

Morasso, P. (1981). Spatial control of arm movements. Experimental Brain Research, 42:223-227.

Nagasaki, H. (1989). Asymmetric velocity profiles and acceleration profiles of human arm movements. Experimental Brain Research, 74:319-326.

Nelson, W. (1983). Physical principles for economies of skilled movements. Biological Cybernetics, 46:135-147.

Ohta, K., Svinin, M., Luo, Z., Hosoe, S., and Laboissière, R. (2004). Optimal trajectory formation of constrained human arm reaching movements. Biological Cybernetics, 91(1):2336.

Piazzi, A. and Visioli, A. (2000). Minimum-time system-inversion-based motion planning for residual vibration reduction. IEEE/ASME Transactions on Mechatronics, 5(1):12-22.

Prudnikov, A., Brychkov, Y., and Marichev, O. (1986). Integrals and Series, Vol. 1: Elementary Functions. Gordon \& Breach, New York.

Richardson, M. and Flash, T. (2002). Comparing smooth arm movements with the two-thirds power law and the related segmented-control hypothesis. The Journal of Neuroscience, 22(18):8201-8211.

Rose, N. and Bronson, R. (1969). On optimal terminal control. IEEE Transactions on Automatic Control, 14(5):443-449.

Samko, S., Kilbas, A., and Marichev, Y. (1993). Integrals and Derivatives of Fractional Order and Their Applications. Gordon and Breach Science Publications. 
Soechting, J. and Lacquaniti, F. (1981). Invariant characteristics of a pointing movement in man. The Journal of Neuroscience, 1:710-Ü720.

Stein, R., Oguztoreli, M., and Capaday, C. (1986). What is optimized in muscular movements? In Jones, N., McCartney, M., and McComas, A., editors, Human Muscle Power, pages 131Ü-150. Human Kinetics, Champaign, Illinois.

Svinin, M., Goncharenko, I., and Hosoe, S. (2008). On the boundary conditions in modeling of human-like reaching movements. In Proc. IEEE/RSJ Int. Conference on Intelligent Robots and Systems, IROS'2008, volume 1, pages 518-525, Nice, France.

Svinin, M., Masui, Y., Luo, Z., and Hosoe, S. (2005). On the dynamic version of the minimum hand jerk criterion. Journal of Robotic Systems, 22(11):661-676.

Tsuji, T., Tanaka, Y., Morasso, P., Sanguineti, V., and Kaneko, M. (2002). Bio-mimetic trajectory generation of robots via artificial potential field with time base generator. IEEE Transactions on Systems, Man, and Cybernetics-Part C: Applications and Reviews, 32(4):426439.

Uno, Y., Kawato, M., and Suzuki, R. (1989). Formation and control of optimal trajectory in human multijoint arm movement. minimum torque-change model. Biological Cybernetics, 61:89-101.

Wiegner, A. and Wierbicka, M. (1992). Kinematic models and elbow flexion movements: Quantitative analysis. Experimental Brain Research, 88:665-673. 


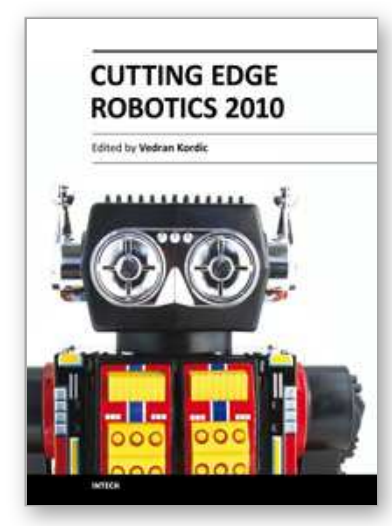

\author{
Cutting Edge Robotics 2010 \\ Edited by Vedran Kordic
}

ISBN 978-953-307-062-9

Hard cover, 440 pages

Publisher InTech

Published online 01, September, 2010

Published in print edition September, 2010

Robotics research, especially mobile robotics is a young field. Its roots include many engineering and scientific disciplines from mechanical, electrical and electronics engineering to computer, cognitive and social sciences. Each of this parent fields is exciting in its own way and has its share in different books. This book is a result of inspirations and contributions from many researchers worldwide. It presents a collection of a wide range of research results in robotics scientific community. We hope you will enjoy reading the book as much as we have enjoyed bringing it together for you.

\title{
How to reference
}

In order to correctly reference this scholarly work, feel free to copy and paste the following:

Mikhail M. Svinin, Igor A. Goncharenko, Shigeyuki Hosoe and Yoshihito Osada (2010). Optimality Principles and Motion Planning of Human-Like Reaching Movements, Cutting Edge Robotics 2010, Vedran Kordic (Ed.), ISBN: 978-953-307-062-9, InTech, Available from: http://www.intechopen.com/books/cutting-edge-robotics2010/optimality-principles-and-motion-planning-of-human-like-reaching-movements

\section{INTECH}

open science / open minds

\section{InTech Europe}

University Campus STeP Ri

Slavka Krautzeka 83/A

51000 Rijeka, Croatia

Phone: +385 (51) 770447

Fax: +385 (51) 686166

www.intechopen.com

\section{InTech China}

Unit 405, Office Block, Hotel Equatorial Shanghai

No.65, Yan An Road (West), Shanghai, 200040, China

中国上海市延安西路65号上海国际贵都大饭店办公楼405单元

Phone: +86-21-62489820

Fax: +86-21-62489821 
(C) 2010 The Author(s). Licensee IntechOpen. This chapter is distributed under the terms of the Creative Commons Attribution-NonCommercialShareAlike-3.0 License, which permits use, distribution and reproduction for non-commercial purposes, provided the original is properly cited and derivative works building on this content are distributed under the same license. 